MATHEMATICS OF COMPUTATION

Volume 73, Number 247, Pages 1195-1201

S 0025-5718(03)01584-9

Article electronically published on July 14, 2003

\title{
PIECEWISE LINEAR FINITE ELEMENT METHODS ARE NOT LOCALIZED
}

\author{
ALAN DEMLOW
}

\begin{abstract}
Recent results of Schatz show that standard Galerkin finite element methods employing piecewise polynomial elements of degree two and higher to approximate solutions to elliptic boundary value problems are localized in the sense that the global dependence of pointwise errors is of higher order than the overall order of the error. These results do not indicate that such localization occurs when piecewise linear elements are used. We show via simple one-dimensional examples that Schatz's estimates are sharp in that localization indeed does not occur when piecewise linear elements are used.
\end{abstract}

\section{INTRODUCTION}

In [Sch98, Schatz introduced global weighted maximum norm error estimates for the standard Galerkin finite element method for the second-order linear elliptic Neumann problem

$$
\begin{aligned}
-\sum_{i, j=1}^{N} \frac{\partial}{\partial x_{j}}\left(a_{i j}(x) \frac{\partial u}{\partial x_{i}}\right)+\sum_{i=1}^{N} b_{i}(x) \frac{\partial u}{\partial x_{i}}+c(x) u & =f & \text { in } \Omega, \\
\frac{\partial u}{\partial n_{L}} & =0 & \text { on } \partial \Omega,
\end{aligned}
$$

where $\frac{\partial u}{\partial n_{L}}$ is the conormal derivative and $\Omega$ is a domain in $\mathbb{R}^{N}$ with smooth boundary $\partial \Omega$. While the pointwise dependence of the error in the finite element method upon $u$ and its derivatives is in general global, these weighted estimates show that, for all finite element spaces of polynomial order two and higher, standard Galerkin finite element methods are localized in the sense that the global dependence of the pointwise error occurs only via higher order terms. However, Schatz's results indicate (but do not prove) that no localization occurs in the lowest-order piecewise linear case. The purpose of this note is to investigate the sharpness of this result.

Asymptotic error expansion inequalities were given in [Sch98] as elementary consequences of the weighted maximum norm estimates proved therein. These expansions are convenient for use in applications (cf. [HSWW01]) and display localization properties more clearly than do the weighted maximum norm estimates they are derived from, so we shall only state the expansions. We also note that the sharpness of the expansions implies sharpness of the weighted norm results since the former are derived from the latter. We let $u_{h} \in S_{h}^{r}$ be the Galerkin approximation to a solution $u$ to (1.1). Here $r$ is the order of approximation given by $S_{h}^{r}$ in $L_{p}$

Received by the editor July 22, 2002 and, in revised form, December 15, 2002.

2000 Mathematics Subject Classification. Primary 65N30, 65N15.

This material is based upon work supported under a National Science Foundation graduate fellowship and under NSF grant DMS-0071412. 
$(1 \leq p \leq \infty)$ and $h$ is taken to be the diameter of an element in a globally quasiuniform mesh. Schatz proved that for $r \geq 3$ (i.e., for piecewise quadratic elements and higher),

$$
\left(u-u_{h}\right)(x) \leq C\left(\sum_{r \leq|\alpha| \leq 2 r-3} h^{|\alpha|}\left|D^{\alpha} u(x)\right|+h^{2 r-2-\epsilon}\|u\|_{W_{\infty}^{2 r-2}(\Omega)}\right),
$$

where $\epsilon>0$. We note that (1.2) in fact shows that higher order polynomial approximating spaces lead to higher degrees of localization in that the order $2 r-2-\epsilon$ of the global term increases relative to the order of approximation $r$ given by $S_{h}^{r}$ as $r$ increases. This fact is not important for our further purposes, however, so we also record here the simplified (but not sharp, except when $r=3$ ) estimate

$$
\left|\left(u-u_{h}\right)(x)\right| \leq C\left(\sum_{|\alpha|=r} h^{r}\left|D^{\alpha} u(x)\right|+h^{r+1-\epsilon}\|u\|_{W_{\infty}^{r+1}(\Omega)}\right) .
$$

For first derivatives, Schatz showed that for $r \geq 2$ (i.e., for piecewise linear elements and higher),

$$
\left|\nabla\left(u-u_{h}\right)(x)\right| \leq C\left(\sum_{r \leq|\alpha| \leq 2 r-2} h^{|\alpha|-1}\left|D^{\alpha} u(x)\right|+h^{2 r-2-\epsilon}\|u\|_{W_{\infty}^{2 r-1}(\Omega)}\right) .
$$

As above, one may instead derive a simplified expansion of the form

$$
\left|\nabla\left(u-u_{h}\right)(x)\right| \leq C\left(h^{r-1} \sum_{|\alpha|=r}\left|D^{\alpha} u(x)\right|+h^{r-\epsilon}\|u\|_{W_{\infty}^{r+1}(\Omega)}\right) .
$$

Besides having inherent theoretical interest, these estimates have been applied in HSWW01 to the analysis of asymptotically exact a posteriori error estimators. However, this analysis requires localization of the pointwise error $\left(u-u_{h}\right)(x)$, thus excluding the lowest-order piecewise linear case $r=2$. An analysis of such estimators for the piecewise linear case was given by Schatz and Wahlbin in [SW02, but this required a different argument. This application and general theoretical interest lead us to consider whether an expansion of the form (1.3) is possible in the piecewise linear case, that is, whether

$$
\left|\left(u-u_{h}\right)(x)\right| \leq C\left(h^{2} \sum_{|\alpha|=2}\left|D^{\alpha} u(x)\right|+h^{2+\delta}\|u\|_{W_{\infty}^{3}(\Omega)}\right)
$$

holds for any $\delta>0$. We demonstrate via a simple one-dimensional example that the estimate (1.6) in fact does not hold for any $\delta>0$, that is, standard piecewise linear finite element methods for elliptic problems are not localized. We also show that the estimate (1.5) for first derivatives is sharp with respect to localization when $r=2$ in the sense that an estimate of the form

$$
\left|\nabla\left(u-u_{h}\right)(x)\right| \leq C\left(h \sum_{|\alpha|=2}\left|D^{\alpha} u(x)\right|+h^{2} \sum_{|\alpha|=3}\left|D^{\alpha} u(x)\right|+h^{2+\delta}\|u\|_{W_{\infty}^{4}(\Omega)}\right)
$$

does not hold for any $\delta>0$.

We finally comment on our treatment of logarithmic factors. It is possible to state the estimates (1.3), (1.4), (1.5), and (1.6) without the presence of the small term $\epsilon$ in the exponent of the global terms at the expense of a factor of $\log \frac{1}{h}$ multiplying the right-hand side of each estimate. However, these estimates were applied in the form used above in [HSWW01], and we find them to be clearer in this form as well. We note that our examples still demonstrate the sharpness of 
Schatz's weighted maximum norm estimates and error expansion inequalities with respect to localization even if logarithmic terms were used in their statements.

\section{Results AND PROOFs}

In this section we give our results and their proofs.

2.1. Preliminaries and notation. We shall consider the two-point boundary value problem

$$
-u^{\prime \prime}+u=f \quad \text { in }(0,1)
$$

with either homogeneous Neumann boundary conditions $\left(u^{\prime}(0)=u^{\prime}(1)=0\right)$ or homogeneous Dirichlet conditions $(u(0)=u(1)=0)$. We subdivide the interval $I=(0,1)$ into $n$ subintervals of length $h=\frac{1}{n}$ and denote by $x_{i}$ the meshpoints $i h$, $i=0, \ldots, n$. We denote by $P L_{h}$ the functions which are continuous on $\bar{I}=[0,1]$ and which are linear on each subinterval $\left[x_{i}, x_{i+1}\right]$. In the case of Dirichlet conditions, we require $\chi \in P L_{h}$ to satisfy $\chi(0)=\chi(1)=0$. Given a continuous function $u$, we let $I_{h} u$ be the function in $P L_{h}$ satisfying $I_{h} u\left(x_{i}\right)=u\left(x_{i}\right), i=0, \ldots, n$. The Galerkin approximation $u_{h}$ to $u$ is the unique function in $P L_{h}$ satisfying

$$
\left(u_{h}^{\prime}, v_{h}^{\prime}\right)+\left(u_{h}, v_{h}\right)=\left(u^{\prime}, v_{h}^{\prime}\right)+\left(u, v_{h}\right)=\left(f, v_{h}\right)
$$

for all $v_{h} \in P L_{h}$. Here $(\cdot, \cdot)$ denotes the $L_{2}$ inner product on $I$.

In our results and proofs we shall make use of the Green's function $G(x, y)=$ $G^{x}(y)$ for problem (2.1) (with appropriate boundary conditions) and its Galerkin approximation $G_{h}(x, y)=G_{h}^{x}(y)$. $G^{x}$ satisfies

$$
u(x)=\left(\frac{\partial}{\partial y} G^{x}, u^{\prime}\right)+\left(G^{x}, u\right)
$$

whenever $u$ satisfies appropriate boundary conditions. Similarly, $G_{h}^{x}(y)$ satisfies

$$
\chi(x)=\left(\frac{\partial}{\partial y} G_{h}^{x}, \chi^{\prime}\right)+\left(G_{h}^{x}, \chi\right)
$$

for all $\chi \in P L_{h}$.

\subsection{Statement of results.}

Theorem 2.1. Let $u$ satisfy (2.1) with either homogenous Dirichlet or homogeneous Neumann boundary conditions, and let $u_{h}$ satisfy (2.2). Then

$$
\left(u-u_{h}\right)(x)=\left(u-I_{h} u\right)(x)+\frac{h^{2}}{12} \int_{0}^{1} G^{x}(y) u^{\prime \prime}(y) d y+R_{h}^{1}(u),
$$

and if $x \in\left[x_{i}+\frac{h}{4}, x_{i+1}-\frac{h}{4}\right]$ (i.e., if $x$ lies in the middle half of a mesh interval),

$$
\left(u-u_{h}\right)^{\prime}(x)=\left(u-I_{h} u\right)^{\prime}(x)+\frac{h^{2}}{12} \int_{0}^{1} \frac{\partial}{\partial x} G^{x}(y) u^{\prime \prime}(y) d y+R_{h}^{2}(u),
$$

where $R_{h}^{i}(u) \leq C h^{3}\|u\|_{W_{\infty}^{3}(I)}, i=1,2$.

Our goal is to use the identities (2.5) and (2.6) to show that the errors $\left(u-u_{h}\right)(x)$ and $\left(u-u_{h}\right)^{\prime}(x)$ have a global dependence of order $h^{2}$ upon $u^{\prime \prime}$. We note that the Green's function $G^{x}(y)$ is continuous, positive, increasing for $y<x$, and decreasing for $y>x$. Given these properties and given a fixed point $x \in I$, one may construct functions $u_{1}$ and $u_{2}$ satisfying the desired boundary conditions such that $u_{i}^{\prime \prime}(y)=0$ for $y$ near $x$ (say, for $|x-y|<1 / 8$ ) and such that $M_{1}=\left|\int_{0}^{1} G^{x}(y) u_{1}^{\prime \prime}(y) d y\right|$ and 
$M_{2}=\left|\int_{0}^{1} \frac{\partial}{\partial x} G^{x}(y) u_{2}^{\prime \prime}(y) d y\right|$ are nonzero. If (1.6) and (1.7) were true, then the inequalities

$$
\left|\left(u_{1}-u_{1 h}\right)(x)\right| \leq C h^{2+\delta}\left\|u_{1}\right\|_{W_{\infty}^{3}(I)}=O\left(h^{2+\delta}\right)
$$

and

$$
\left|\left(u_{2}-u_{2 h}\right)^{\prime}(x)\right| \leq C h^{2+\delta}\left\|u_{2}\right\|_{W_{\infty}^{4}(I)}=O\left(h^{2+\delta}\right)
$$

would hold for some $\delta>0$. However, if we apply (2.5) and (2.6) with $h$ small enough, we find that

$$
\begin{aligned}
\left|\left(u_{1}-u_{1 h}\right)(x)\right| & =\left|\frac{h^{2}}{12} \int_{0}^{1} G^{x}(y) u_{1}^{\prime \prime}(y) d y+R_{h}^{1}\left(u_{1}\right)\right| \\
& \geq \frac{h^{2}}{12} M_{1}-C h^{3}\left\|u_{1}\right\|_{W_{\infty}^{3}(I)}=h^{2}\left(\frac{M_{1}}{12}-C h\right) \geq C h^{2}
\end{aligned}
$$

and

$$
\begin{aligned}
\left|\left(u_{2}-u_{2 h}\right)^{\prime}(x)\right| & =\left|\frac{h^{2}}{12} \int_{0}^{1} \frac{\partial}{\partial x} G^{x}(y) u_{2}^{\prime \prime}(y) d y+R_{h}^{2}\left(u_{2}\right)\right| \\
& \geq \frac{h^{2}}{12} M_{2}-C h^{3}\left\|u_{2}\right\|_{W_{\infty}^{3}(I)}=h^{2}\left(\frac{M_{2}}{12}-C h\right) \geq C h^{2},
\end{aligned}
$$

which contradict (2.7) and (2.8), respectively. Thus (1.6) and (1.7) do not hold for any positive choice of $\delta$, and the asymptotic inequalities (and therefore also the weighted maximum norm estimates) derived by Schatz are sharp in the piecewise linear case.

Remark 2.2. In [BLR86], asymptotic error expansion equalities were proved for the standard piecewise linear finite element method with uniform mesh for convex polygonal or smooth domains in $\mathbb{R}^{2}$. These expansions, which are valid for the errors in point values but not for derivatives, have a form similar to that obtained in (2.5), although the expansions in the two-dimensional case depend upon mesh geometry and are thus somewhat more complicated. These results also confirm the sharpness of Schatz's results for errors in point values in the piecewise linear case.

2.3. Proof of Theorem 2.1. We first record a few facts which we shall use in the course of our proofs and then we prove Theorem 2.1

Lemma 2.3. Let $G$ and $G_{h}$ be as defined above. Then

$$
\begin{aligned}
\left\|G^{x}(\cdot)-G_{h}^{x}(\cdot)\right\|_{L_{1}(I)} & \leq C h^{2}, \\
\left\|G(x, \cdot)-G_{h}(x, \cdot)\right\|_{W_{1}^{1}(I)} & \leq C h .
\end{aligned}
$$

Let $\bar{x}_{i}=\frac{x_{i}+x_{i+1}}{2}$. Then if $x \in\left[x_{i}, x_{i+1}\right]$,

$$
\left(u-I_{h} u\right)(x)=\frac{1}{2} u^{\prime \prime}\left(\bar{x}_{i}\right)\left(x-x_{i}\right)\left(x-x_{i+1}\right)+O\left(h^{3}\right),
$$

where $O\left(h^{3}\right)$ denotes a term bounded by $C h^{3}\|u\|_{W_{\infty}^{3}(I)}$. Finally, the Galerkin approximation to the Green's function is piecewise linear and continuous in $x$ as well as $y$. That is, $G_{h}(x, y)$ is a continuous piecewise bilinear function on $\bar{I} \times \bar{I}$.

Proof. We first note that given standard maximum-norm results for finite element methods in one dimension such as those given in [Whe73, (2.9) and (2.10) may be proven via a duality argument; (2.11) is proved via an elementary argument involving Taylor expansions. The final fact, i.e., that $G_{h}(x, y)$ is continuous and 
piecewise linear in $x$ as well as in $y$, may be verified as follows. Let $\left\{\psi_{1}, \ldots, \psi_{M}\right\}$ be the basis for $P L_{h}$ satisfying $\psi_{i}\left(x_{j}\right)=\delta_{i j}$, and let $S$ be the matrix corresponding to the finite element equations (2.2) relative to the basis $\left\{\psi_{1}, \ldots, \psi_{M}\right\}$. Finally, let $\vec{V}(z):[0,1] \rightarrow \mathbb{R}^{M}$ be the vector function with $\psi_{i}(z)$ as its $i$ th entry. Then it is easy to compute that $G_{h}(x, y)=(\vec{V}(y))^{T} S^{-1} \vec{V}(x)$, which is a continuous piecewise bilinear function.

Proof of Theorem 2.1. We first note that throughout the course of our proofs, we shall denote by $O\left(h^{k}\right)$ any term bounded by $C h^{k}\|u\|_{W_{\infty}^{3}(I)}$. We begin our proof by noting that

$$
\left(u-u_{h}\right)(x)=\left(u-I_{h} u\right)(x)+\left(I_{h} u-u_{h}\right)(x) .
$$

Using the definition of $G_{h}^{x}$ and (2.2), we next find that

$$
\begin{aligned}
\left(I_{h} u-u_{h}\right)(x) & =\left(\left(I_{h} u-u_{h}\right)^{\prime}, \frac{\partial}{\partial y} G_{h}^{x}\right)+\left(I_{h} u-u_{h}, G_{h}^{x}\right) \\
& =\left(\left(I_{h} u-u\right)^{\prime}, \frac{\partial}{\partial y} G_{h}^{x}\right)+\left(I_{h} u-u, G_{h}^{x}\right) .
\end{aligned}
$$

We next note that $\frac{\partial}{\partial y} G_{h}^{x}$ is constant on each element $\left[x_{i}, x_{i+1}\right]$ and $\left(I_{h} u-u\right)\left(x_{i}\right)=0$ for all $i=0, \ldots, n$ so that $\left(I_{h} u-u\right)^{\prime}$ has a zero average on each interval $\left[x_{i}, x_{i+1}\right]$. Thus we deduce that

$$
\begin{gathered}
\left(\left(I_{h} u-u\right)^{\prime}, \frac{\partial}{\partial y} G_{h}^{x}\right)=\int_{0}^{1}\left(I_{h} u-u\right)^{\prime}(y) \frac{\partial}{\partial y} G_{h}^{x}(y) d y \\
=\sum_{i=0}^{n-1} \int_{x_{i}}^{x_{i+1}}\left(I_{h} u-u\right)^{\prime}(y) \frac{\partial}{\partial y} G_{h}(x, y) d y=0 .
\end{gathered}
$$

We then insert (2.14) into (2.13) to find that

$$
\left(I_{h} u-u_{h}\right)(x)=\left(I_{h} u-u, G_{h}^{x}\right)=\left(I_{h} u-u, G_{h}^{x}-G^{x}\right)+\left(I_{h} u-u, G^{x}\right) .
$$

Employing (2.9) and approximation theory for piecewise linear functions yields

$$
\left(I_{h} u-u, G_{h}^{x}-G^{x}\right) \leq C\left\|I_{h} u-u\right\|_{L_{\infty}(I)}\left\|G_{h}^{x}-G^{x}\right\|_{L_{1}(I)} \leq O\left(h^{4}\right) .
$$

Thus

$$
\left(u-u_{h}\right)(x)=\left(u-I_{h} u\right)(x)+\int_{0}^{1}\left(I_{h} u-u\right)(y) G^{x}(y) d y+O\left(h^{4}\right) .
$$

Then using (2.11) and employing the midpoint rule several times, we deduce that

$$
\begin{aligned}
\int_{0}^{1}\left(I_{h} u-u\right)(y) G^{x}(y) d y \\
\quad=-\frac{1}{2} \sum_{i=0}^{n-1} \int_{x_{i}}^{x_{i+1}} u^{\prime \prime}\left(\bar{x}_{i}\right)\left(y-x_{i}\right)\left(y-x_{i+1}\right) G^{x}\left(\bar{x}_{i}\right) d y+O\left(h^{3}\right) \\
\quad=\frac{h^{2}}{12} \int_{0}^{1} u^{\prime \prime}(y) G^{x}(y) d y+O\left(h^{3}\right) .
\end{aligned}
$$

We complete the proof of (2.5) by inserting (2.18) into (2.17). 
In order to prove (2.6), we differentiate both sides of (2.15) with respect to $x$ to find that for $x \in\left[x_{i}+\frac{h}{4}, x_{i+1}-\frac{h}{4}\right]$,

$$
\begin{aligned}
\left(I_{h} u\right. & \left.-u_{h}\right)^{\prime}(x)=\int_{0}^{x}\left(I_{h} u-u\right)(y) \frac{\partial}{\partial x}\left(G_{h}(x, y)-G(x, y)\right) d y \\
& +\int_{x}^{1}\left(I_{h} u-u\right)(y) \frac{\partial}{\partial x}\left(G_{h}(x, y)-G(x, y)\right) d y \\
& +\int_{0}^{1}\left(I_{h} u-u\right)(y) \frac{\partial}{\partial x} G(x, y) d y .
\end{aligned}
$$

We note that differentiating under the integrals in the above equation is permissible because $G(x, y)$ is smooth (with bounded derivatives of all orders) except along the line $y=x$, and $G_{h}(x, y)$ is a continuous piecewise bilinear function. We next use Taylor's theorem while noting that $\frac{\partial^{2}}{\partial x^{2}} G_{h}(x, y)=0$ inside of each mesh interval to find that, for $y<x$,

$$
\begin{aligned}
{\left[G\left(x_{i+1}, y\right)-G_{h}\left(x_{i+1}, y\right)\right] } & \\
=\left[G(x, y)-G_{h}(x, y)\right]+ & {\left[\frac{\partial}{\partial x}\left(G(x, y)-G_{h}(x, y)\right)\right]\left(x_{i+1}-x\right) } \\
& +\frac{1}{2} \frac{\partial^{2}}{\partial x^{2}} G(\xi, y)\left(x_{i+1}-x\right)^{2}
\end{aligned}
$$

for some $\xi \in\left(x, x_{i+1}\right)$. We solve (2.20) for $\frac{\partial}{\partial x}\left(G(x, y)-G_{h}(x, y)\right)$, then apply approximation theory and (2.9) while recalling that $\frac{\partial^{2}}{\partial x^{2}} G(x, y)$ is bounded on $[0,1] \times$ $[0,1] \backslash\{x=y\}$ and $\frac{1}{x_{i+1}-x} \leq \frac{4}{h}$ to find

$$
\begin{aligned}
& \int_{0}^{x}\left(I_{h} u-u\right)(y) \frac{\partial}{\partial x}\left(G_{h}(x, y)-G(x, y)\right) d y \\
& \leq\left\|I_{h} u-u\right\|_{L_{\infty}(0, x)}\left\|\frac{\partial}{\partial x}\left(G_{h}(x, \cdot)-G(x, \cdot)\right)\right\|_{L_{1}(0, x)} \\
& \leq O\left(h^{2}\right) \frac{4}{h}\left[\left\|G^{x_{i+1}}-G_{h}^{x_{i+1}}\right\|_{L_{1}(0, x)}+\left\|G^{x}-G_{h}^{x}\right\|_{L_{1}(0, x)}\right. \\
& \left.\quad+h^{2} \int_{0}^{x}\left\|\frac{\partial^{2}}{\partial x^{2}} G(\cdot, y)\right\|_{L_{\infty}\left(x, x_{i+1}\right)} d y\right] \\
& \quad \leq O\left(h^{3}\right) .
\end{aligned}
$$

We may employ the same argument with $x_{i}$ in place of $x_{i+1}$ to find that

$$
\int_{x}^{1}\left(I_{h} u-u\right)(y) \frac{\partial}{\partial x}\left(G_{h}(x, y)-G(x, y)\right) d y \leq O\left(h^{3}\right) .
$$

We may finally compute exactly as in (2.18) that

$$
\int_{0}^{1}\left(I_{h} u-u\right)(y) \frac{\partial}{\partial x} G(x, y) d y=\frac{h^{2}}{12} \int_{0}^{1} \frac{\partial}{\partial x} G^{x}(y) u^{\prime \prime}(y) d y+O\left(h^{3}\right) .
$$

Combining (2.19), (2.21), (2.22), and (2.23) completes the proof of (2.6) and thus of Theorem 2.1 . 


\section{ACKNOWLEDGMENTS}

I would like to thank Bob Strichartz for asking the questions which originally motivated these results and Lars Wahlbin and Al Schatz for their comments regarding this note.

\section{REFERENCES}

[BLR86] Heribert Blum, Qun Lin, and Rolf Rannacher, Asymptotic error expansion and Richardson extrapolation for linear finite elements, Numer. Math. 49 (1986), 11-37. MR 87m:65172

[HSWW01] Wolfgang Hoffmann, Alfred H. Schatz, Lars B. Wahlbin, and Gabriel Wittum, Asymptotically exact a posteriori estimators for the pointwise gradient error on each element in irregular meshes. I. A smooth problem and globally quasi-uniform meshes, Math. Comp. 70 (2001), no. 235, 897-909. MR 2002a:65178

[Sch98] Alfred H. Schatz, Pointwise error estimates and asymptotic error expansion inequalities for the finite element method on irregular grids. I. Global estimates, Math. Comp. 67 (1998), no. 223, 877-899. MR 98j:65082

[SW02] Alfred H. Schatz and Lars B. Wahlbin, Asymptotically exact a posteriori estimators for the pointwise gradient error on each element in irregular meshes. Part II: The piecewise linear case, to appear.

[Whe73] Mary Fanett Wheeler, An optimal $L_{\infty}$ error estimate for Galerkin approximations to solutions of two-point boundary value problems, SIAM J. Numer. Anal. 10 (1973), 914-917. MR 49:8399

Department of Mathematics, Malott Hall, Cornell University, Ithaca, New York 14853

E-mail address: ard11@cornell.edu 